PHYSICAL REVIEW B 84, 195420 (2011)

\title{
Molecular dynamics study of the thermopower of Ag, Au, and Pt nanocontacts
}

\author{
F. Pauly, ${ }^{1,}$ J. K. Viljas,,${ }^{2,3}$ M. Bürkle, ${ }^{1}$ M. Dreher, ${ }^{4}$ P. Nielaba, ${ }^{4}$ and J. C. Cuevas ${ }^{5}$ \\ ${ }^{1}$ Institut für Theoretische Festkörperphysik and DFG Center for Functional Nanostructures, Karlsruhe Institute of Technology, \\ D-76131 Karlsruhe, Germany \\ ${ }^{2}$ Low Temperature Laboratory, Aalto University, P.O. Box 15100, FIN-00076 Aalto, Finland \\ ${ }^{3}$ Department of Physics, P.O. Box 3000, FIN-90014 University of Oulu, Finland \\ ${ }^{4}$ Fachbereich Physik, Universität Konstanz, D-78457 Konstanz, Germany \\ ${ }^{5}$ Departamento de Física Teórica de la Materia Condensada, Universidad Autónoma de Madrid, E-28049 Madrid, Spain
}

(Received 7 July 2011; revised manuscript received 13 October 2011; published 4 November 2011)

\begin{abstract}
Using molecular dynamics simulations of many junction stretching processes combined with tight-bindingbased electronic structure and transport calculations, we analyze the thermopower of silver (Ag), gold (Au), and platinum (Pt) atomic contacts. In all cases we observe that the thermopower vanishes on average within the standard deviation and that its fluctuations increase for a decreasing minimum cross section of the junctions. However, we find a suppression of the fluctuations of the thermopower for the $s$-valent metals $\mathrm{Ag}$ and $\mathrm{Au}$, when the conductance originates from a single, perfectly transmitting channel. Essential features of the experimental results for $\mathrm{Au}, \mathrm{Ag}$, and copper $(\mathrm{Cu})$ of Ludoph and van Ruitenbeek [Phys. Rev. B 59, 12290 (1999)], as yet unaddressed by atomistic studies, can hence be explained by considering the atomic and electronic structure at the disordered narrowest constriction of the contacts. For the multivalent metal $\mathrm{Pt}$ our calculations predict the fluctuations of the thermopower to be larger by one order of magnitude as compared to $\mathrm{Ag}$ and $\mathrm{Au}$, and suppressions of the fluctuations as a function of the conductance are absent. Main features of our results are explained in terms of an extended single-level model.
\end{abstract}

DOI: 10.1103/PhysRevB.84.195420

\section{INTRODUCTION}

The development of thermoelectric devices for the efficient conversion of heat into electrical energy or for refrigeration would be an important step toward a more environmentally friendly use of energy. Engineered nanostructures are promising materials in this respect, ${ }^{1-4}$ and molecular junctions are presently moving into the focus of research..$^{5-7}$ They seem to be good candidates for achieving high thermoelectric figures of merit as a result of the discrete energy level structure of the molecules ${ }^{5,8}$ and because molecular properties can be controlled by chemical synthesis. Already the measurement of the thermopower alone provides important insights into the electronic structure of molecular junctions, not accessible by conductance measurements. ${ }^{9}$ Thus, electron and hole conduction can be distinguished ${ }^{10}$ and the alignment of molecular levels with respect to the Fermi energy can be determined. ${ }^{9,11,12}$ It turns out that the experiments are described by a combination of electronic structure and transport calculations. ${ }^{13,14}$ For instance, the linear increase of the thermopower with molecule length in the typical off-resonant transport situation has been found in both experiment ${ }^{9,12,15}$ and theory. ${ }^{13,16,17}$

While molecular junctions appear promising from a deviceoriented point of view, metallic electrodes are typically used to contact the molecules. ${ }^{9,11,12}$ Metallic atomic contacts hence constitute important reference systems. Until now their thermopower has been studied experimentally in the singleatom contact regime only by Ludoph and van Ruitenbeek. ${ }^{18}$ The authors discuss mostly their results for Au contacts, but measurements for $\mathrm{Ag}$ and $\mathrm{Cu}$ contacts have been performed as well. ${ }^{18,19}$

Motivated by pioneering experiments with high-mobility two-dimensional electron gases, ${ }^{20,21}$ theoretical studies have described the thermopower of quantum point contacts in two
PACS number(s): 73.63.Rt, 72.15.Jf, 73.23.Ad, 72.10.Fk

and three dimensions using the free-electron-gas approximation for adiabatic constrictions. ${ }^{21-24}$ Indeed, such studies have successfully predicted maxima of the thermopower at the transition between quantized conductance plateaus. ${ }^{20,22}$ The Fermi wavelength of several $10 \mathrm{~nm}$ for the two-dimensional electron gases (see Ref. 25, for instance) is much larger than the atomic dimensions. For metals, in contrast, the Fermi wavelength is on the order of the interatomic distance. ${ }^{10}$ Therefore, disorder-related effects due to the atomic structure are expected to play a more important role than for the two-dimensional electron gas devices, and material-specific chemical properties enter as an additional aspect. To describe the metallic atomic contacts, it is hence necessary to take into account both the atomic and the detailed electronic structure. $^{26-33}$

Recent theoretical studies of the thermopower of nanocontacts have considered separately the effects of disorder and material-specific parameters. Thus, thermoelectric effects for structures with atomically thin one-dimensional wires connected to two-dimensional electrodes were studied in Refs. 34 and 35 for various geometries and with disorder, but without material-specific parameters. In contrast in Refs. 36 and 37 material-specific parameters were employed, while crucial disorder-related effects, resulting in the statistical nature of the experiments, ${ }^{18}$ were neglected by treating only ideal wire geometries.

In this paper we present fully atomistic simulations of the thermopower of atomic contacts for three different metals, namely $\mathrm{Ag}, \mathrm{Au}$, and Pt. The methods we use are essentially the same as employed by us to study the conductance histograms of atomic contacts of various metals. ${ }^{31,32}$ The junction geometries are obtained by molecular dynamics (MD) simulations of many junction stretching processes. The determination of the electronic structure is based on the Slater-Koster ${ }^{38}$ 
tight-binding (TB) parameters of Papaconstantopoulos et al $^{39,40}$ that take into account $s$-, $p$-, and $d$-type atomic orbitals, thus allowing for a fairly realistic description of metals. For the transport calculations we employ the LandauerBüttiker formalism combined with standard Green's-function methods.

The results for the monovalent metals, especially $\mathrm{Au}$, are compared to the available experimental data, ${ }^{18}$ while predictions are made for the multivalent Pt. In all cases we observe that the thermopower fluctuates between positive and negative values, such that the average essentially vanishes compared to the standard deviation. It is also found that the fluctuations are larger in the narrower junctions. However, there are also important differences. For the $s$-valent metals the thermopower fluctuations are found to be strongly suppressed close to a conductance of $1 G_{0}$, where $G_{0}=2 e^{2} / h$ is the quantum of conductance, while for Pt no such suppression is predicted. This is because in the $s$-valent metals the conductance then tends to originate from a single, perfectly transmitting channel, while for multivalent metals several partially open channels are involved. The suppression of the fluctuations for $\mathrm{Au}$ and $\mathrm{Ag}$ is consistent with the experimental results of Ludoph and van Ruitenbeek ${ }^{18}$ for $\mathrm{Au}, \mathrm{Ag}$, and $\mathrm{Cu}$. The experiments can hence be explained by considering the atomic and electronic structure at the disordered narrowest constriction of the contacts. As another difference between the $s$-valent and multivalent metals, our calculations predict the thermopower fluctuations for $\mathrm{Pt}$ to be larger by one order of magnitude as compared to $\mathrm{Ag}$ and $\mathrm{Au}$.

In addition to the fully atomistic "hybrid" MD/TB-transport calculations we introduce a simple "extended single-level model" (ESLM) to better understand the main features of the simulation results. This model is compared with a related model introduced in Ref. 18.

The manuscript is organized as follows. In Sec. II we present the methodological details of our atomistic simulations and then discuss the results in Sec. III. In Sec. III we also introduce the ESLM. Conclusions and an outlook are given in Sec. IV. In Appendix A some further examples of junction stretching processes are shown, Appendix B discusses specifics related to the eigenchannel transmission properties, and Appendix $\mathrm{C}$ presents the details of the ESLM. Animations of junction stretching processes are provided as electronic Supplemental Material. $^{41}$

\section{THEORETICAL APPROACH}

Our calculations proceed along the lines of Refs. 31 and 32, which we extend here to study the thermopower. The calculations can be divided essentially into two parts, namely (i) the generation of contact geometries and (ii) the determination of electronic structure and transport properties.

\section{A. Molecular dynamics simulations}

The contact geometries used in this work for $\mathrm{Ag}, \mathrm{Au}$, and Pt are those employed in our previous studies. ${ }^{31,32}$ For the sake of completeness, we describe their construction briefly.

We determine contact geometries by performing classical MD simulations of junction stretching processes. As displayed

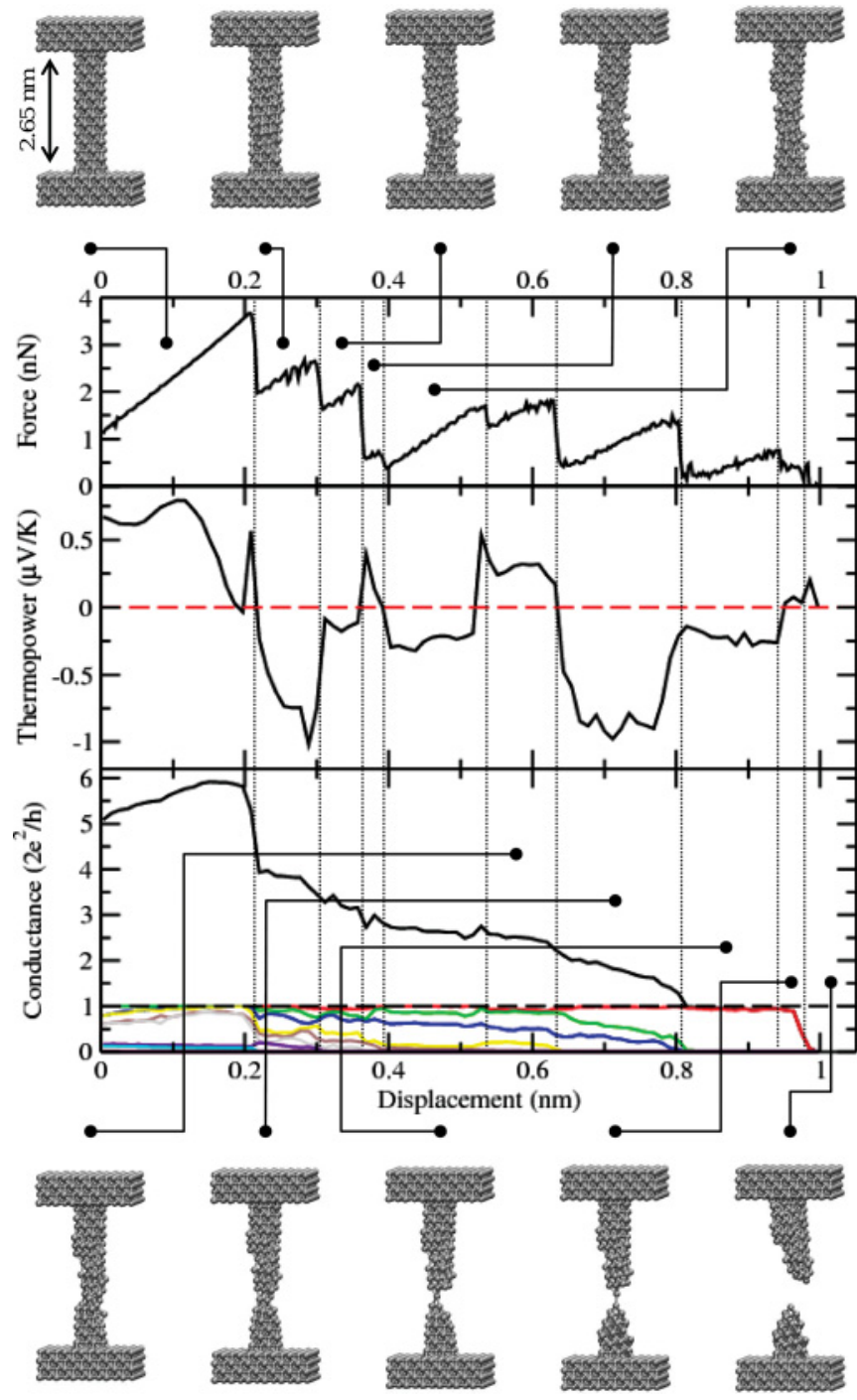

FIG. 1. (Color online) Formation of an Ag dimer contact. The upper, middle, and lower panels show, respectively, the tensile force, the thermopower, and the conductance as a function of the electrode displacement. For the conductance also the decomposition into conduction eigenchannels $G_{0} \tau_{n}$ is displayed. Horizontal dashed lines indicate the zero level of the thermopower or a conductance of $1 G_{0}$, while the vertical dotted lines mark the main plastic reorganizations of the contact. Above and below the graphs snapshots of the contact geometry during the stretching process are shown, and the length of the central wire is given for zero electrode displacement.

in Figs. 1-3, we choose nanowire geometries with a central wire $(\mathrm{CW})$ connected to larger-diameter electrodes which are attached at the top and bottom. Initially, we assume all atoms to be located at the positions of a perfect fcc lattice, with the $\langle 100\rangle$ direction oriented along the $z$ axis. The lattice constants are determined by minimizing the potential energy of a crystal. When we use the semiempirical potentials of our MD calculations, ${ }^{42}$ we obtain lattice constants of 0.408 , $0.406,0.393 \mathrm{~nm}$ for $\mathrm{Ag}, \mathrm{Au}, \mathrm{Pt}$, respectively, which are close to the experimental values. ${ }^{10}$ The CW consists of 112 atoms with 14 layers along the $z$ direction and eight atoms per layer. Its initial length amounts to $2.65,2.64,2.55 \mathrm{~nm}$ 


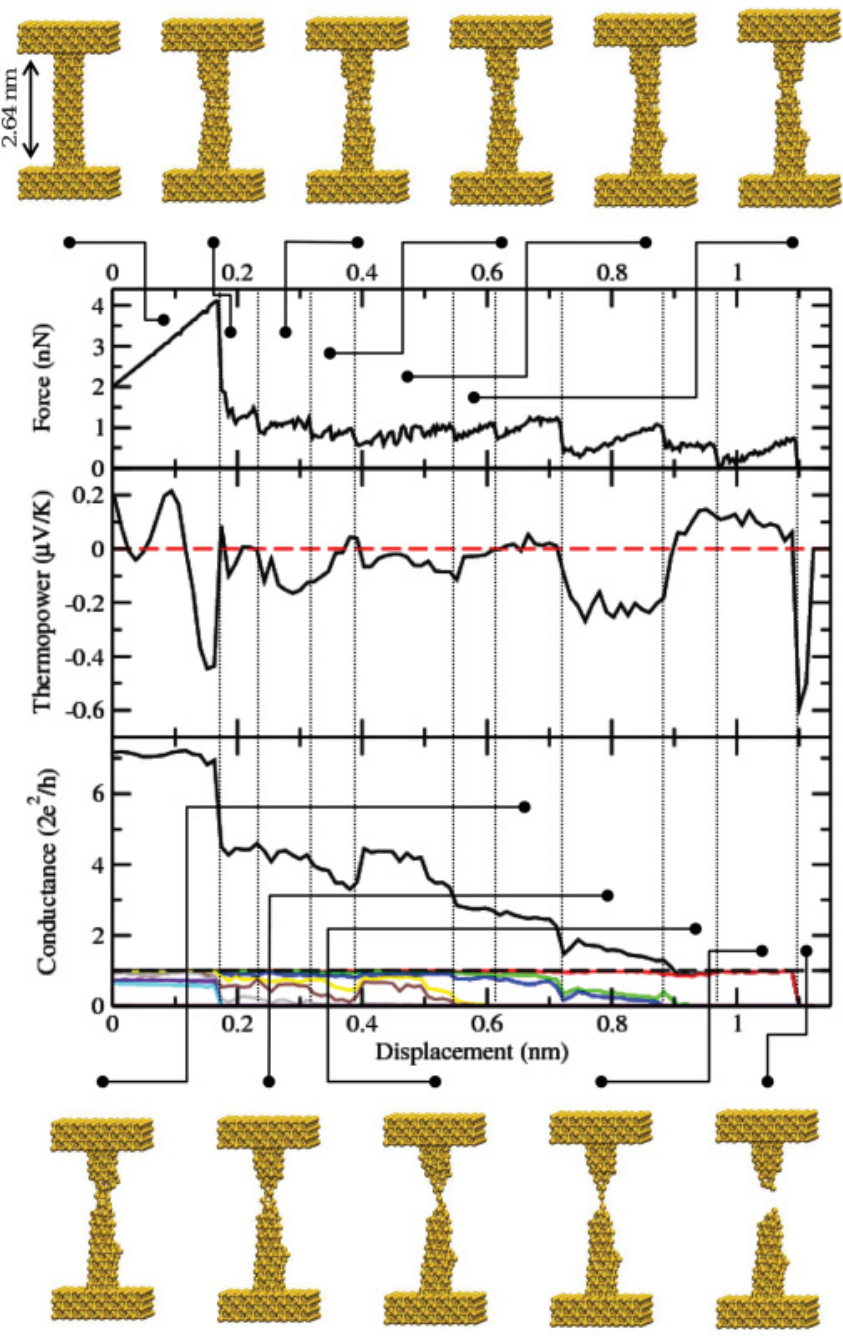

FIG. 2. (Color online) The same as Fig. 1, but for the formation of an $\mathrm{Au}$ dimer contact.

for $\mathrm{Ag}, \mathrm{Au}, \mathrm{Pt}$, respectively, as indicated in Figs. 1-3. In each case the electrodes at the top and bottom contain 288 atoms.

Junction stretching is performed by separating the otherwise fixed electrodes symmetrically by a constant distance during every time step of $1.4 \mathrm{fs}$. In this process, we use periodic boundary conditions along the $z$ direction and the minimum image convention for the supercells perpendicular to it. ${ }^{43}$ The constant stretching speed amounts to $2 \mathrm{~m} / \mathrm{s}$. The forces on the wire atoms are calculated from semiempirical potentials, ${ }^{42}$ while their average temperature remains at $4.2 \mathrm{~K}$ by use of a Nosé-Hover thermostat. ${ }^{44}$ Different junction evolutions are obtained by choosing random starting velocities for the atoms in the CW. Every 1.4 ps a contact geometry is recorded, and the strain force is calculated. ${ }^{45}$

\section{B. Electronic structure and transport properties}

Transport properties are determined within the LandauerBüttiker formalism. ${ }^{13,21,31-33,46}$ The conductance $G$ and thermopower $S$ are expressed as

$$
G=G_{0} K_{0}(T),
$$
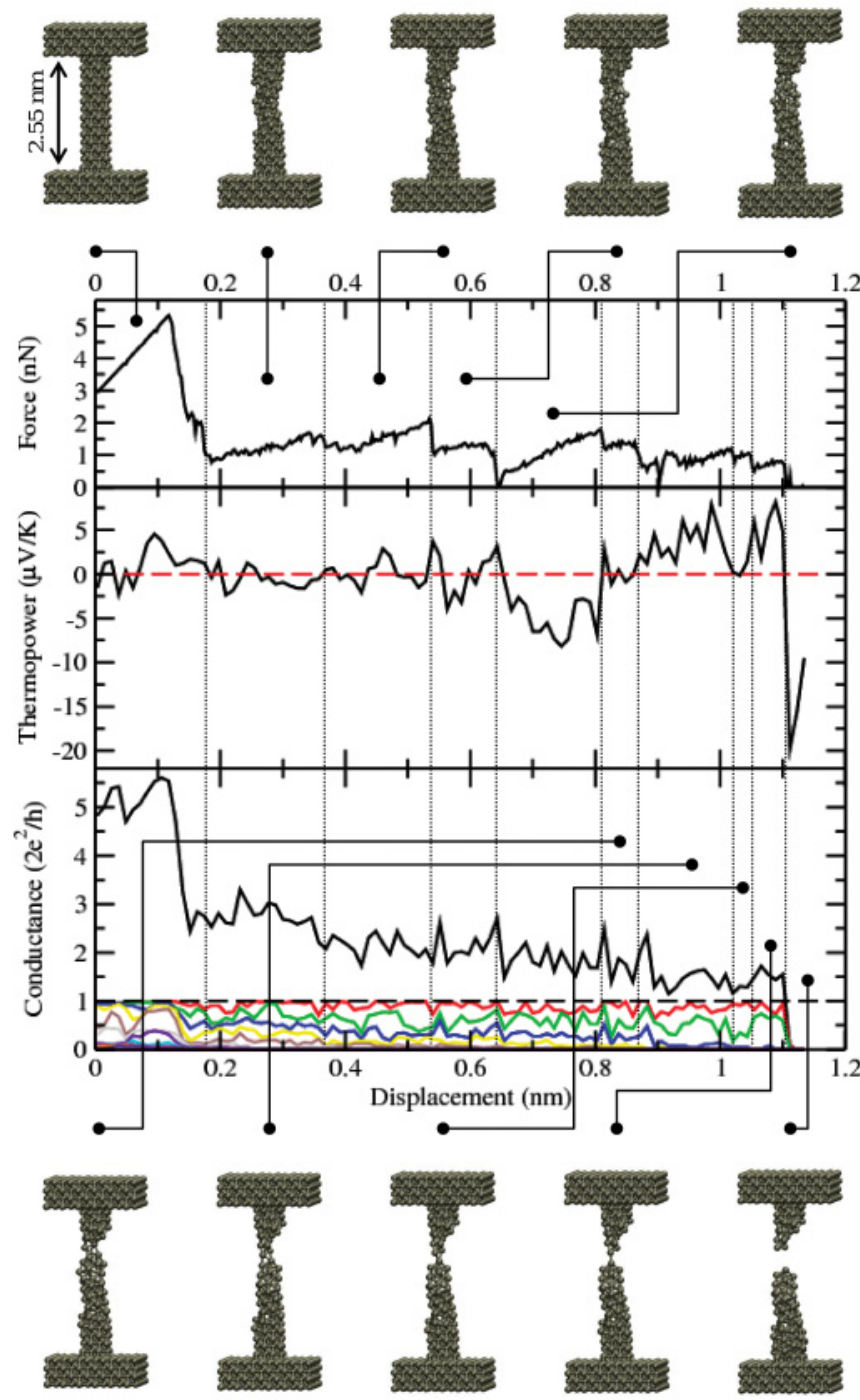

FIG. 3. (Color online) The same as Fig. 1, but for the formation of a Pt dimer contact.

$$
S=-\frac{K_{1}(T)}{e T K_{0}(T)},
$$

with $K_{n}(T)=\int d E(E-\mu)^{n} \tau(E)\left[-\partial_{E} f(E, T)\right]$, the absolute value of the electron charge $e=|e|$, the transmission function $\tau(E)$, the Fermi function $f(E, T)=\{\exp [(E-$ $\left.\left.\mu) / k_{B} T\right]+1\right\}^{-1}$, the Boltzmann constant $k_{B}$, and the electrochemical potential $\mu$, which approximately equals the metal Fermi energy $\mu \approx E_{F}$.

In order to compare to the experiments of Ref. 18, we assume in the following a temperature of $T=12 \mathrm{~K}$ for the determination of the transport properties. While we evaluate $S$ via Eq. (2), for the conductance the simpler low-temperature formula,

$$
G=G_{0} \tau\left(E_{F}\right)=G_{0} \sum_{n} \tau_{n}\left(E_{F}\right),
$$

yields a good approximation. Here, $\tau$ has been resolved into the contributions of transmission probabilities $\tau_{n}$ from the individual transmission eigenchannels $n$. As discussed below, 
they provide important information for the understanding of the results.

We obtain the transmission and the channel decomposition by use of Green's-function techniques, as described in Ref. 32. We assume the central device region to be equal to the $\mathrm{CW}$, while the remaining atoms of the contacts are attributed to the electrodes. However, while in Ref. 32 we assumed the unperturbed electrode Green's functions to be those of the bulk, in the present study we use surface Green's functions. They are determined via a decimation technique by modeling the electrodes as surfaces of ideal, semi-infinite crystals. ${ }^{33,47}$ Even though the results for these two different procedures differ only slightly, ${ }^{48}$ surface Green's functions describe the physical situation more accurately.

To perform the energy integrations in Eq. (2), we have computed $\tau(E)$ every 5.6 ps for 11 equally spaced points in the energy interval $\left[E_{F}-\Delta, E_{F}+\Delta\right]$ with $\Delta=$ $0.05 \mathrm{eV} . K_{n}(T)$ is then obtained by the integration of $(E-\mu)^{n} \tau(E)\left[-\partial_{E} f(E, T)\right]$, using a linear interpolation for $\tau(E)$ between the energy sampling points.

The effective single-particle Hamiltonian and overlap matrices needed for the evaluation of the transmission are obtained from a Slater-Koster ${ }^{38}$ TB description, ${ }^{39,40}$ supplemented by a local charge neutrality condition. ${ }^{13}$ Even if this approach is not at the level of self-consistent $a b$ initio methods, it is still atomistic and takes into account the symmetries of the $s, p$, and $d$ valence orbitals for these monoatomic systems. In the past, it was used successfully to describe the conduction properties of various metallic atomic contacts. ${ }^{31,32,49-51}$

\section{RESULTS AND DISCUSSION}

\section{A. Junction stretching events}

In order to analyze the behavior of the thermopower for the metallic atomic contacts, we have simulated 50 stretching processes for each of the $\mathrm{Ag}, \mathrm{Au}$, and Pt nanowires. Beside the thermopower we have analyzed the strain force and the conductance with its decomposition into individual channel contributions. Examples of stretching events, which we will discuss in the following paragraphs, are shown in Figs. 1-3. In all the selected cases the contacts break after a dimer contact has formed (i.e., a two-atom chain). Further examples of stretching events, including the formation of longer atomic chains for $\mathrm{Au}$ and $\mathrm{Pt}$ contacts, are provided in Appendix A.

The evolution of the mechanical and transport properties for an Ag contact are displayed in Fig. 1. From the strain force, shown in the upper panel of the figure, elastic and plastic stages can be distinguished. While the force increases in a linear manner in the elastic ones, it drops suddenly when bonds break and atoms rearrange during the short plastic stages. ${ }^{52}$ Similar to what is shown in the experimental plots for $\mathrm{Au}$ in Ref. 18, the thermopower in the middle panel behaves in a step-wise manner. It takes both positive and negative values, and fluctuates around zero. As visible from the dotted lines in Fig. 1, the steps in the thermopower typically coincide with plastic deformations of the contact with smooth changes in between. All these features and also the absolute values of the thermopower are in agreement with the experimental results. We note that while data are presented in Ref. 18 only for $\mathrm{Au}$, it is stated there that studies of $\mathrm{Ag}$ and $\mathrm{Cu}$ samples showed similar results, which justifies our comparison. The conductance is displayed in the lowest panel of Fig. 1. After an initial increase, it drops in a gradual manner. Before contact rupture it decreases from a value of around $2 G_{0}$ to a clear plateau at $1 G_{0}$, when a single atom is at the narrowest constriction. The contact breaks quickly after a dimer has formed. For the single-atom and dimer configurations the current is carried by a single fully transmitting channel, as expected for the $s$-valent metals. ${ }^{27,28,31,32,53}$

The evolution of junction properties for an Au contact is shown in Fig. 2. As suggested by the comparable valence electronic structure of the atoms, the results resemble those of Ag. The thermopower behaves in a step-wise fashion and exhibits gradual changes or plateaus during the elastic stages, interrupted by the plastic deformations. Again it takes both positive and negative values, and the thermopower is comparable in magnitude to those of $\mathrm{Ag}$ and the data presented in Ref. 18. The conductance falls with a revival to a value of $1.9 G_{0}$. It decreases then in a rather continuous way to a value of $1.3 G_{0}$ before it drops to a value slightly below $1 G_{0}$, carried by a single channel. The conductance changes only little, when the single-atom contact transforms into the rather stable dimer contact.

For multivalent Pt the situation is expected to change, and an example is shown in Fig. 3. We observe that also here the thermopower fluctuates around zero, but with absolute values roughly one order of magnitude larger as compared to Ag and $\mathrm{Au}$. The occurrence of these strong fluctuations, seen also in the conductance, is due to the important contribution of $d$ states at $E_{F}$. They give rise to a pronounced energy dependence of the transmission especially for the narrow contacts, as discussed in Ref. 32. Also, due to the higher anisotropy of these states as compared to the spherically symmetric $s$-valence orbitals of $\mathrm{Ag}$ and $\mathrm{Au}$ the junction transport properties exhibit a high sensitivity to changes in the atomic positions. ${ }^{32}$ While we are not aware of any measurements of the thermopower for atomic $\mathrm{Pt}$ contacts, we observe that the conductance for the singleatom and dimer contacts is at around $1.5 G_{0}$ (see the electrode displacements above $1.02 \mathrm{~nm}$ ). The multivalent electronic structure and hence the contribution of several channels to the transmission at $E_{F}$ typically lead to conductance values above $1 G_{0}$ for one-atom-wide constrictions, in agreement with the interpretation of measured opening traces and conductance histograms. ${ }^{27,54-56}$

\section{B. Statistical analysis of atomistic simulations}

Collecting the data of many stretching processes, we perform a statistical analysis similar to the experiments. ${ }^{18}$ For this purpose, we use the 50 simulated opening events of the nanowires for each metal. Typically the contacts break via the single atom (Ag, 12\%; $\mathrm{Au}, 8 \% ; \mathrm{Pt}, 4 \%$ ) or dimer (Ag, 82\%; $\mathrm{Au}, 68 \%$; $\mathrm{Pt}, 60 \%$ ) configurations mentioned before, with a single atom or a chain of two atoms in the narrowest part, respectively. As visible from the percentage of occurrence, the dimers are generally preferred. However, we find also atomic chain geometries with chain lengths of three atoms or more (Ag, 6\%; $\mathrm{Au}, 24 \% ; \mathrm{Pt}, 36 \%$ ). For Ag such structures are rare. 


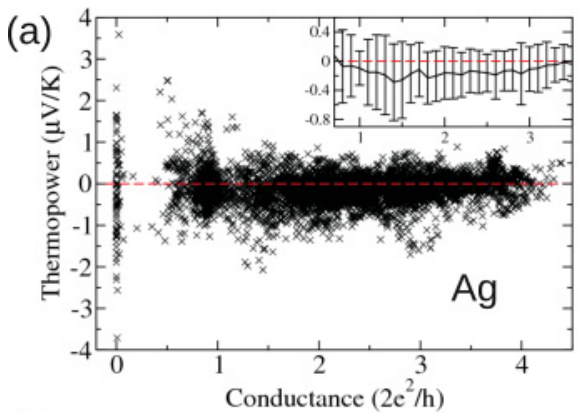

(d)

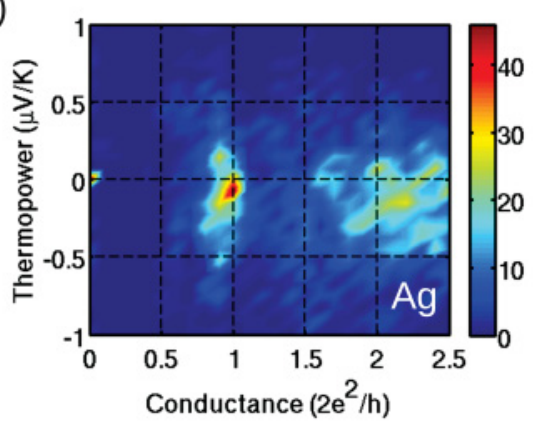

(b)

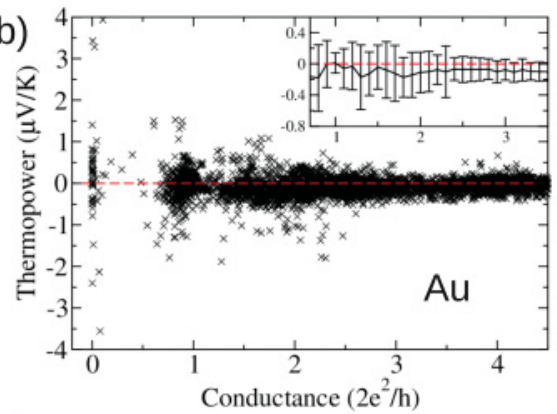

(e)

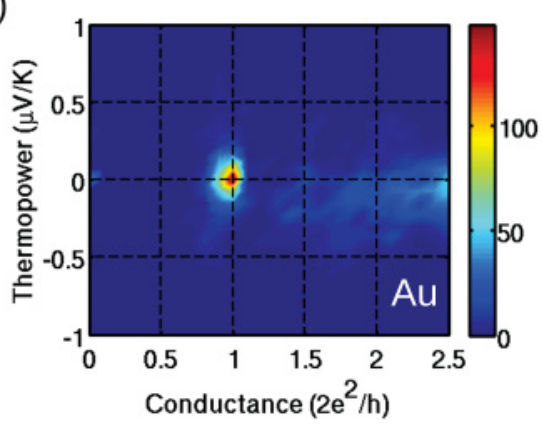

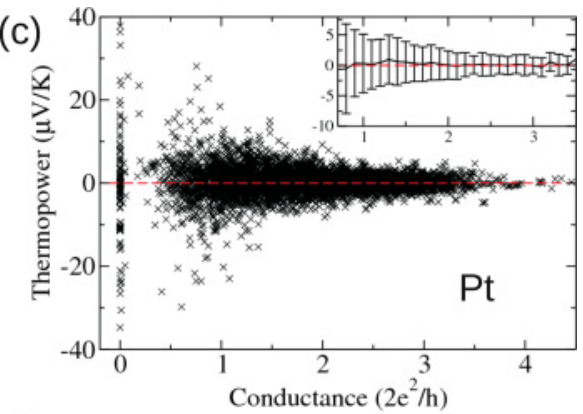

(f)

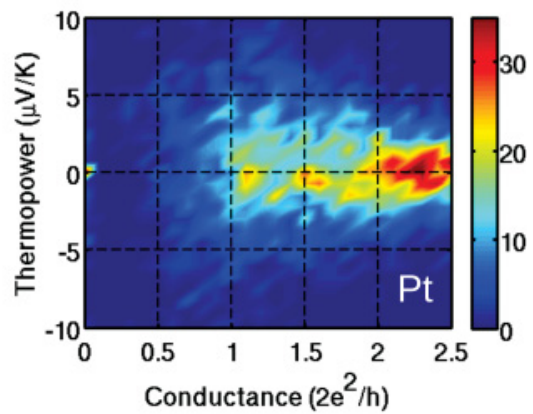

FIG. 4. (Color online) Scatter plots of the thermopower as a function of the conductance for (a) Ag, (b) Au, and (c) Pt. The main panels show all computed data points, while the insets display the average of the thermopower together with its standard deviation. Panels (d)-(f) are the corresponding density plots. For the insets of plots (a)-(c) and the density plots (d)-(f), the bin size of the conductance is $0.1 G_{0}$. Those of the thermopower amount to $0.075 \mu \mathrm{V} / \mathrm{K}$ for (d) and (e), and $0.75 \mu \mathrm{V} / \mathrm{K}$ for (f).

Out of the 50 stretching events, we find at rupture only three chain configurations with chain lengths varying from three to five atoms. While such structures are generally believed to be unlikely, ${ }^{57,58}$ they have been observed in transmission electron microscopy studies. ${ }^{59}$ For Au and Pt atomic chains occur more frequently in our simulations, and they are longer. We have decided to exclude the junctions breaking with chains of 10 atoms or more in length from our analysis, since experiments indicate maximum lengths of up to eight atoms. ${ }^{54,60,61}$ For $\mathrm{Au}$ we have additionally excluded an opening process leading to a chain with six atoms, because the thermopower exhibits peculiar features before contact rupture related to $d$ states. For this reason we present below the statistical analysis of the transport properties with 50 stretching events for Ag, 46 for $\mathrm{Au}$, and 41 for $\mathrm{Pt}$.

The thermopower-conductance $(S-G)$ plots are presented in Fig. 4. The main panels of Figs. 4(a)-4(c) show all the computed data points. This representation provides an impression of the overall scatter. In the insets the average value of the thermopower $\langle S\rangle_{G}$ and the standard deviation $\sigma_{S}=\sqrt{\left\langle S^{2}\right\rangle_{G}-\langle S\rangle_{G}^{2}}$ are plotted. While $\langle S\rangle_{G}$ is very close to zero for $\mathrm{Pt}$, we find a trend toward negative values for $\mathrm{Ag}$ and $\mathrm{Au}$. From the theory of the free-electron gas in a hyperbolic constriction $^{62}$ a thermopower with a unique, negative sign is expected. ${ }^{18,19}$ We cannot exclude such an origin for the trend, but our results with thermopower values of both positive and negative sign demonstrate that it is necessary to go beyond these simple free-electron-gas models with idealized wire geometries to describe even the $s$-valent metallic atomic contacts. Furthermore, within the standard deviation the results for $\langle S\rangle_{G}$ are consistent with a vanishing thermopower. From Figs. 4(a)-4(c) we also observe that the variations of $S$ tend to increase for a decreasing minimum cross section of the contacts. Consistent with the sample opening traces discussed above, the variations of the thermopower around zero are predicted to be one order of magnitude larger for Pt than for Ag and Au.

With respect to $\langle S\rangle_{G} \approx 0$ and increasing $\sigma_{S}$ for decreasing $G$, the $S-G$ scatter plot for Au closely resembles the experimental one of Ref. 18. However, the absolute magnitude of the scatter of $S$ in our calculations is too small by a factor of around 3 , and we will discuss possible reasons for this discrepancy below. ${ }^{18,19}$

In order to reveal further differences between the monoand multivalent metals, we show the corresponding $S-G$ density plots in Figs. 4(d)-4(f). For Ag and Au they exhibit a pronounced maximum at $(G, S)=\left(1 G_{0}, 0\right)$, while there is no particular feature for $\mathrm{Pt}$ at this position. The peak for the $s$ valent metals seems plausible. In the conductance histograms of $\mathrm{Ag}$ and $\mathrm{Au}$ pronounced maxima occur at $1 G_{0} .^{28,54}$ Our calculated conductance histograms, shown in Figs. 5(a) and 5(b), are consistent with this experimental finding. ${ }^{31,32}$ Since the mean thermopower vanishes, maxima at $\left(1 G_{0}, 0\right)$ are thus expected in the density plots of Figs. 4(d) and 4(e). The experimental conductance histogram for Pt shows instead a rather broad distribution between 1 and $2 G_{0}$ with a maximum at around $1.5 G_{0} .^{54-56}$ These features are not reproduced in detail in our calculations for $\mathrm{Pt}$ in Fig. 5(c), which shows a broad distribution of frequently occurring conductances between 1 and $3 G_{0}$ without a clear maximum. Possible reasons for the deviations, such as the limited ensemble of considered junction geometries or the approximations inherent to our method, have been discussed in Ref. 32. Still, from the broad distribution of conductance values no sharp peak feature at a 


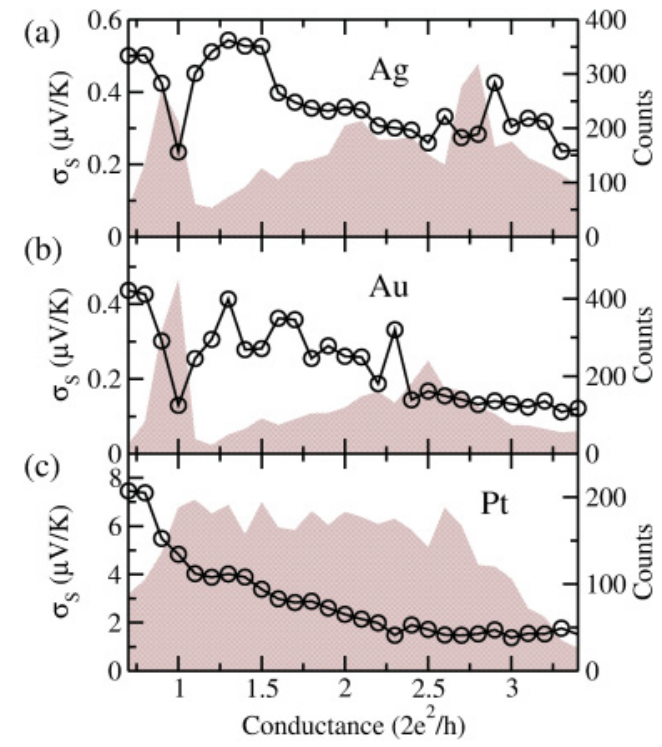

FIG. 5. (Color online) Standard deviation $\sigma_{S}$ of the thermopower as a function of the conductance for (a) Ag, (b) Au, and (c) Pt. Shown in the background is the conductance histogram for each metal, and the number of counts is indicated on the right $y$ axis. In each case the bin size of the conductance is $0.1 G_{0}$.

single conductance value is expected in the $S-G$ density plot, in agreement with Fig. 4(f).

While our discussion of Figs. 4(d)-4(f) considered until now the conductance values, the sharpness of the peak feature at $\left(1 G_{0}, 0\right)$ with respect to the thermopower axis is somewhat unexpected for $\mathrm{Ag}$ and $\mathrm{Au}$. Further insight is obtained by plotting the standard deviation $\sigma_{S}$ as a function of the conductance, as displayed in Fig. 5. The calculations show indeed a suppression of $\sigma_{S}$ at $1 G_{0}$ for $\mathrm{Ag}$ and $\mathrm{Au}$, which explains the sharpness of the feature in the corresponding $S-G$ density plots in Figs. 4(d) and 4(e). Suppressions of $\sigma_{S}$ at higher conductance values are absent in Figs. 5(a) and 5(b), while shallow minima were measured for Au in that region. ${ }^{18}$ Consistent with the underestimation of the scatter of $S$ in Fig. 4(b), $\sigma_{S}$ for $\mathrm{Au}$ is smaller than in the experiments by a factor of 3. For the multivalent metal Pt no particular features arise throughout the whole range of conductance values considered in Fig. 5(c); especially there is no minimum of $\sigma_{S}$ at $1 G_{0}$.

\section{Extended single-level model}

In order to describe in simple terms the behavior of the thermopower fluctuations of the fully atomistic simulations in Fig. 5, we construct an ESLM. We assume that the transmission is given as

$$
\tau(E)=\sum_{n} \tau_{n}\left(E, \epsilon_{0}^{(n)}, \Gamma_{L}^{(n)}, \Gamma_{R}^{(n)}\right),
$$

with

$$
\tau_{n}\left(E, \epsilon_{0}^{(n)}, \Gamma_{L}^{(n)}, \Gamma_{R}^{(n)}\right)=\frac{\Gamma_{L}^{(n)} \Gamma_{R}^{(n)}}{\left(E-\epsilon_{0}^{(n)}\right)^{2}+\frac{\left(\Gamma_{L}^{(n)}+\Gamma_{R}^{(n)}\right)^{2}}{4}} .
$$

The function $\tau_{n}\left(E, \epsilon_{0}^{(n)}, \Gamma_{L}^{(n)}, \Gamma_{R}^{(n)}\right)$ describes a resonance in the energy-dependent transmission probability of the $n$th eigenchannel, which is located close to the Fermi energy. The resonances arise from the atomic level structure at the narrowest part of the contact and quantum interference effects related to the disorder in the CW. Hence, the "level" $\epsilon_{0}^{(n)}$ specifies the position of the resonance for the $n$th eigenchannel, and $\Gamma_{L}^{(n)}, \Gamma_{R}^{(n)}$ determine the width and height of the resonance. For an atomic contact described by $N$ eigenchannels, we can obtain from Eqs. (4) and (5) the conductance and thermopower using the low-temperature expressions of Eqs. (1) and (2) for a given realization of parameters $\epsilon_{0}^{(n)}, \Gamma_{L}^{(n)}, \Gamma_{R}^{(n)}$ with $n=$ $1, \ldots, N$. As explained in detail in Appendices B and C, we identify the $\tau_{n}\left(E_{F}, \epsilon_{0}^{(n)}, \Gamma_{L}^{(n)}, \Gamma_{R}^{(n)}\right)$ with respective eigenchannel transmission probabilities $\tau_{n}$ in order to distinguish between mono- and multivalent metals.

As the simplest model for a monovalent metal we assume that the transmission channels open one by one, that is, that $\tau_{1}=\cdots=\tau_{n-1}=1$ and $\tau_{n}=G / G_{0}-(n-1)$ for $n-1 \leqslant$ $G / G_{0} \leqslant n$. In this case (see Appendix C) we find that

$$
\sigma_{S}=\alpha \sqrt{n-G / G_{0}} / n,
$$

for $G \rightarrow n G_{0}$ in the conductance interval $(n-1) G_{0} \leqslant G \leqslant$ $n G_{0}$, and $\alpha$ is a constant prefactor of dimension $\mathrm{V} / \mathrm{K}$. This relation explains the minimum of $\sigma_{S}$ at $1 G_{0}$ as visible in Figs. 5(a) and 5(b). Minima at higher conductance values $n G_{0}$ with $n>1$ are absent for $\mathrm{Ag}$ and $\mathrm{Au}$, since the assumption that channels open one by one, also known as the "saturation of channel transmission," $18,28,53,63$ is accurately obeyed in our calculations only for the first channel, that is, for conductances below $1 G_{0}$ (see Appendix B and Refs. 31 and 32). The presence of minima at integer conductance values above $1 G_{0}$ is hence hard to understand from our simulations. Frequently occurring junction geometries with several atomic chains in parallel $^{64}$ may provide an explanation, and investigations of junctions with larger initial diameters could help to resolve the puzzle.

In contrast to the monovalent metals the ESLM of a multivalent metal, as presented in Appendix C, predicts that minima of $\sigma_{S}$ are absent, or at least strongly reduced, due to the contribution of several partially open transmission channels throughout the whole range of conductance values. This is consistent with our results from the atomistic simulations in Fig. 5(c).

\section{Discussion}

The behavior of the thermopower has been discussed in terms of a different and very detailed model in Refs. 18 and 28 , which is applicable to the whole range of conductance values. However, apart from the prefactor, at $G$ below but close to $n G_{0}$ their expression for $\sigma_{S}$ in monovalent metals is identical to our Eq. (6). As a common feature, both our and their approach explain the suppression of the thermopower fluctuations by disorder-related quantum interference effects (see also Appendix C). In their model the thermopower fluctuations arise from the interference of directly transmitted electrons and those backscattered elastically in the diffusive regions in the vicinity of the narrowest part of the contact. The backscattering contributions are considered to lowest order, and channel transmissions in the ballistic central region are 
assumed to be energy independent and fixed for a certain $G$. In contrast, in our atomistic simulations we determine the energydependent transmission of the disordered CW, take scattering effects into account to all orders, and different realizations of the set of $\tau_{n}$ may contribute for a given value of the conductance.

In experiments like those of Ref. 18, both local and nonlocal interference contributions will be present and backscattering from defects up to the coherence length of some $100 \mathrm{~nm}$ away from the constriction has been reported at the low temperatures relevant here. ${ }^{65}$ Such highly nonlocal interference contributions are clearly not described by our short CWs. This may result in an underestimation of the energy dependence of the transmission, causing the factor of 3 discrepancy with respect to the experimental values in Figs. 4(b) and 5(b) mentioned above. ${ }^{18}$ Another reason for the discrepancy may be the large ensemble of experimentally realized contact configurations, not fully taken into account in our calculations.

While measurements of the thermopower and its fluctuations are not yet available for Pt, Ludoph and van Ruitenbeek have shown within their model that $\sigma_{S}$ is proportional to the standard deviation $\sigma_{G V}$ of the voltage-dependent conductance. $^{28}$ The comparison of their results for $\sigma_{G V}$ for the transition metals niobium and iron and the $s$-valent metals $\mathrm{Cu}, \mathrm{Ag}$, and $\mathrm{Au}$ shows larger fluctuations by a factor of two for the former. Our prediction of a factor of 10 larger fluctuations for the thermopower of Pt as compared with the $s$-valent metals may be considered as an upper bound, and a reduction might arise from the finite averaging times in the measurements, for instance.

As another important aspect we have discussed only the elastic electronic contribution to the thermopower. Inelastic effects due to the electron-phonon coupling may lead to modifications, which we expect to be small, however, for the experimental conditions considered here. Thus, due to its weakness the electron-phonon coupling can typically be treated perturbatively for metallic atomic contacts, ${ }^{49,61,66,67}$ and the phonon-drag contributions should be suppressed because of the small contact diameter, the low measurement temperatures, and small applied thermal gradients. ${ }^{18,68,69}$ Our results, with the reasonable agreement between experiment and theory, show that the elastic contribution is sufficient to describe the main experimental features.

\section{CONCLUSIONS AND OUTLOOK}

Using molecular dynamics and quantum transport simulations of up to 50 stretching events, we have analyzed the thermopower of atomic contacts of $\mathrm{Ag}, \mathrm{Au}$, and Pt. For $\mathrm{Ag}$ and $\mathrm{Au}$ its behavior agrees well with previously reported measurements. ${ }^{18}$ On a quantitative level, however, the experimental scatter of the thermopower values is underestimated by a factor of around 3. ${ }^{18,19}$ The thermopower-conductance plots show the thermopower to be zero on average within the standard deviation for the three metals studied. Furthermore our calculations predict its variations around the mean to increase for the narrowest constrictions and to be one order of magnitude larger for the multivalent $\mathrm{Pt}$ as compared to the $s$-valent metals Ag and Au. At a conductance of one quantum of conductance we find, in agreement with the experiment, a suppression of the fluctuations of the thermopower for $\mathrm{Ag}$ and $\mathrm{Au}$. For the multivalent metal $\mathrm{Pt}$ possible minima of the fluctuations of the thermopower should be shifted to higher conductance values, but they are predicted to be absent, or at least strongly reduced, due to the influence of several partially open transmission eigenchannels.

Our calculations indicate that the essential characteristics of the thermopower of metallic atomic-size contacts can be understood based on the elastic electronic contribution combined with effects of rather local disorder at the narrowest part of the atomic contacts. Quantitative differences between our simulations and the experiment may arise from the larger variability of contact configurations in the experiments and distant scatterers, not taken into account in the calculations due to the limited system size. ${ }^{28}$ The quantification of effects due to the electron-phonon coupling ${ }^{18,67-69}$ and electron correlations constitute challenging tasks for the future.

\section{ACKNOWLEDGMENTS}

We are grateful to J. M. van Ruitenbeek, E. Scheer, and G. Schön for stimulating discussions. F.P. was funded by the Young Investigator Group and the Center for Functional Nanostructures, and M.B. by the DFG priority program 1243 as well as by the Baden-Württemberg Stiftung within the Network of Excellence "Functional Nanostructures." M.D. and P.N. thank the Collaborative Research Center 767 for funding and the John von Neumann Institute for Computing in Jülich for computer time. J.K.V. acknowledges support from the Academy of Finland, and J.C.C. from the Spanish Ministry of Science and Innovation (Contract No. FIS2008-04209).

\section{APPENDIX A: FURTHER JUNCTION STRETCHING EVENTS}

Here, we provide further examples for the evolution of junction properties upon stretching for each of the three metals studied. For $\mathrm{Au}$ and $\mathrm{Pt}$ we have selected junctions forming chains of several atoms in length before rupture. Animations showing the stretching processes displayed in Figs. 1-3 and 6-8 can be found as Supplemental Material on the Web. ${ }^{41}$

In Fig. 6 we show an Ag contact. After the initial stage, the conductance evolves from a value of around $4 G_{0}$ via various plateaus to around $2 G_{0}$ until a rather stable dimer is formed. For the dimer contact the conductance is pinned closely to $1 G_{0}$ and, at the same time, the thermopower and its fluctuations are suppressed to zero. This particular example agrees well with the observation of the small $\langle S\rangle_{G}$ and minimum of $\sigma_{S}$ for $\mathrm{Ag}$, as shown in Figs. 4 and 5, when the conductance arises from a single completely open conduction channel.

For Au we display in Fig. 7 a contact forming a four-atom chain before breaking. The chain is increasing successively in length, starting from a single atom for electrode displacements between 0.84 and $1.12 \mathrm{~nm}$. Two of them are present from 1.12 to $1.2 \mathrm{~nm}$, three from 1.2 to $1.51 \mathrm{~nm}$, and four from 1.51 to $1.59 \mathrm{~nm}$. As soon as the single-atom contact has formed, 


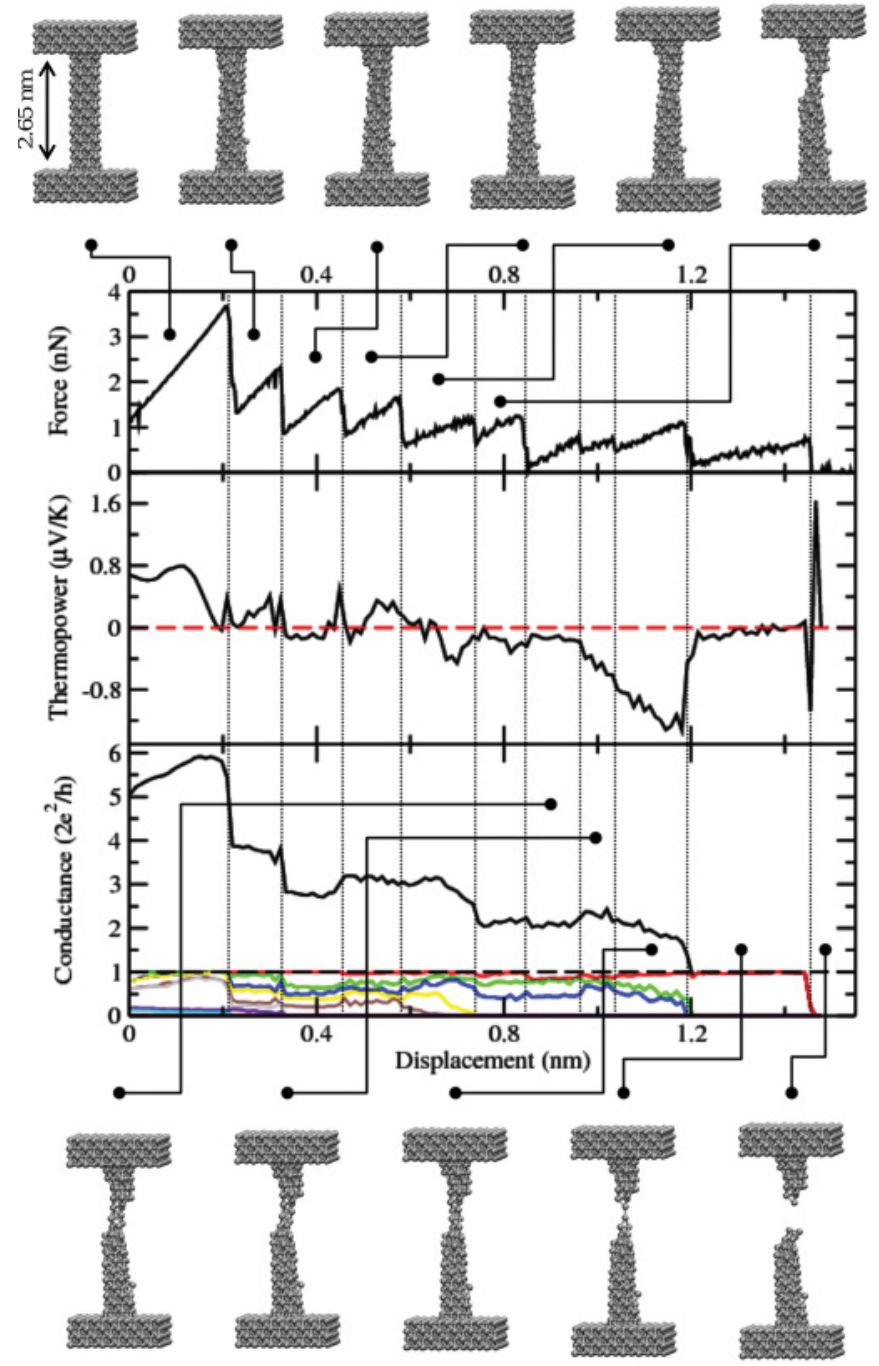

FIG. 6. (Color online) The same as Fig. 1 for another Ag contact forming a dimer before rupture.

only a single channel contributes to the conductance. For the one- to three-atom chain configurations the conductance is very close to $1 G_{0}$ and the thermopower varies around zero with a relatively small amplitude. When the four-atom chain has formed, the conductance is somewhat suppressed and the thermopower increases in absolute magnitude.

In Fig. 8 we show a Pt contact forming a chain of six atoms before rupture. As in Fig. 3 the conductance and thermopower during the stretching show much larger variations than for $\mathrm{Ag}$ and $\mathrm{Au}$, and also the typical values of the thermopower are around one order of magnitude larger than for the $s$-valent metals. After the single-atom contact has formed at around $0.66 \mathrm{~nm}$, more atoms are incorporated into the chain at the elongations marked with dotted lines (i.e., at $0.95,1.11,1.36$, 1.38 , and $1.59 \mathrm{~nm}$ ), until the final length of six atoms is reached. In this atomic-chain regime we generally observe two to three partially open transmission channels, often leading to conductances exceeding $1 G_{0}$. In addition, for these narrowest contacts the thermopower shows its largest fluctuations with the average remaining close to zero.
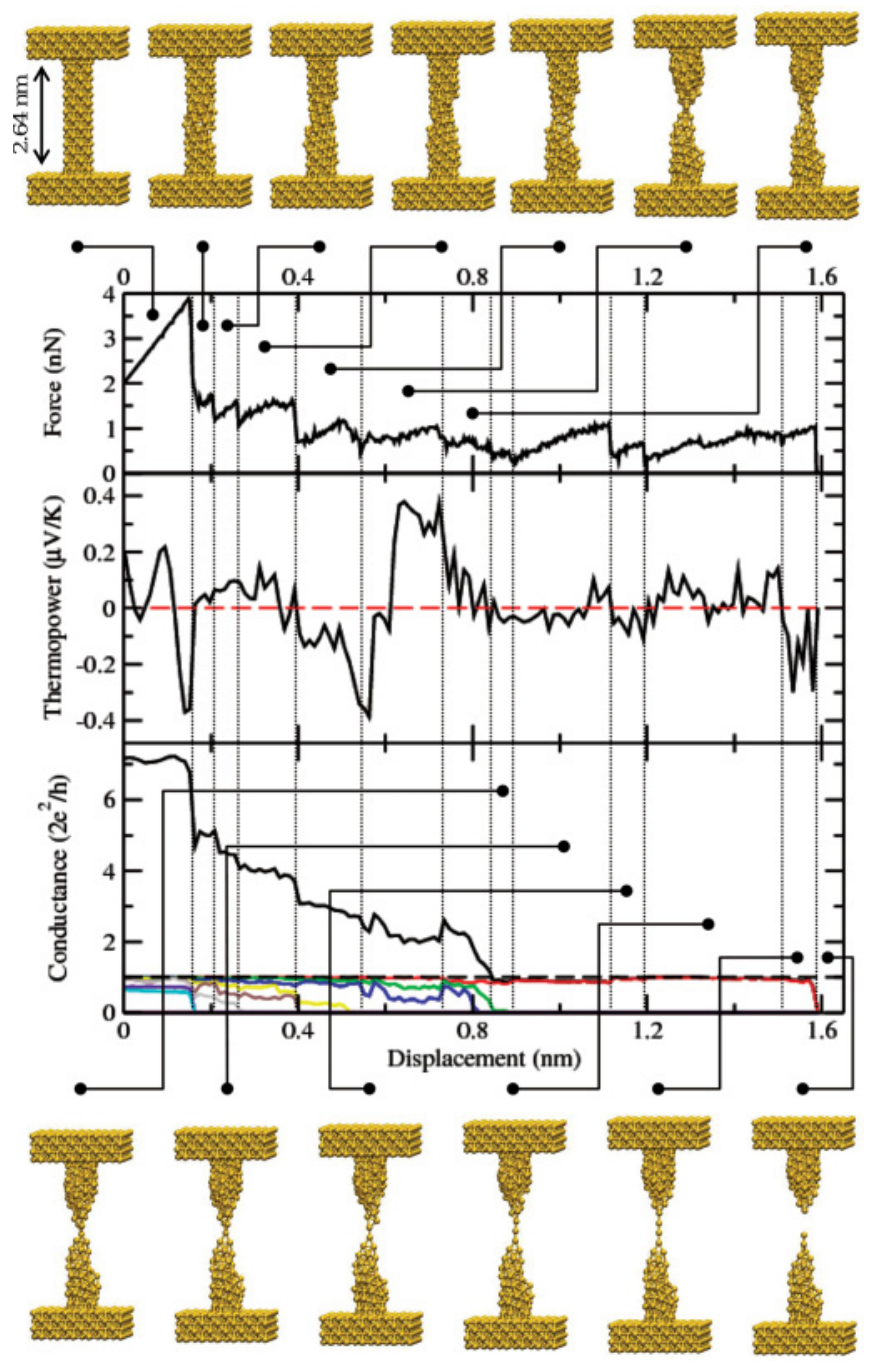

FIG. 7. (Color online) The same as Fig. 1, but for a Au contact forming a chain of four atoms before rupture.

\section{APPENDIX B: AVERAGE EIGENCHANNEL TRANSMISSION PROBABILITIES}

For the monovalent metals transmission eigenchannels are typically assumed to open one by one, while for multivalent metals there are several channels contributing already at the lowest conductances. ${ }^{28,53,63}$ We can inspect the validity of these assertions by examining the average eigenchannel transmission probabilities obtained from our atomistic MD/TB-transport simulations as a function of the junction conductance. ${ }^{31,32}$

Using the results of the present study, we display the average eigenchannel transmissions in Fig. 9. We do indeed observe that for the monovalent metals there is essentially only a single open channel for $0 \leqslant G \leqslant 1 G_{0}$. However, the first channel is not fully open at $1 G_{0}$ (i.e., does not reach a transmission of one). For $G \geqslant 1 G_{0}$ the saturation of channel transmission still seems to be a reasonable approximation for Au. However, the results for $\mathrm{Ag}$ rather suggest an interpretation in terms 


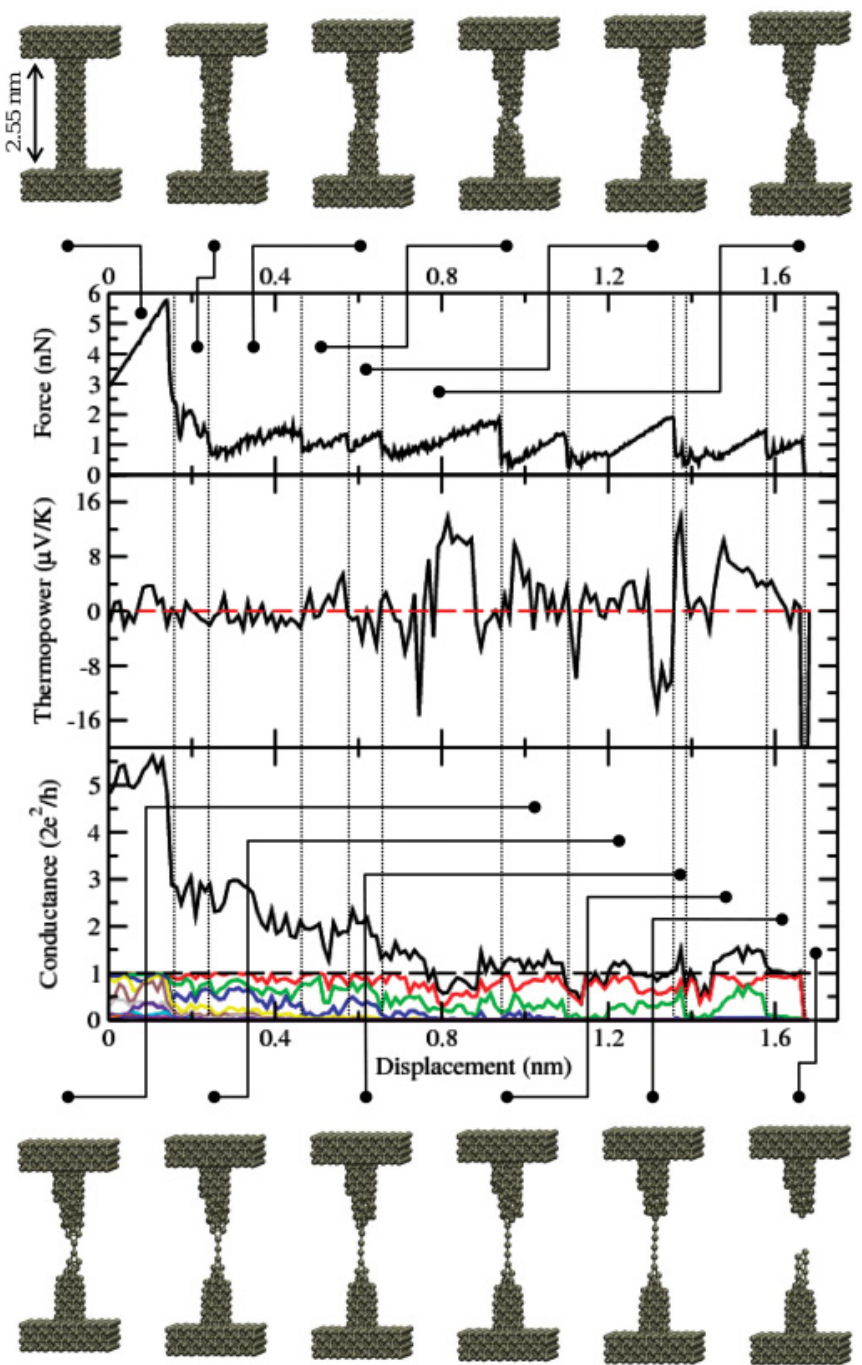

FIG. 8. (Color online) The same as Fig. 1, but for a Pt contact forming a chain of six atoms before rupture.

of a simultaneous opening of two $\pi$-like channels, whose degeneracy is lifted in the disordered junction geometries. For Pt there are significant contributions from at least two partially open channels throughout the whole range of conductance values.

\section{APPENDIX C: FLUCTUATIONS OF THE THERMOPOWER WITHIN THE EXTENDED SINGLE-LEVEL MODEL}

\section{Extended single-level model}

We consider a model for the charge transport through metallic atomic contacts in order to explain the results of our atomistic simulations in simple terms. We assume that the energy-dependent transmission $\tau(E)$ arises from those of independent single-level transmissions, as described by Eqs. (4) and (5). Setting the Fermi energy to zero, $E_{F}=0$, we can determine the conductance and thermopower in the limit of low temperatures as

$$
\begin{aligned}
G & =\left.G_{0} \tau(E)\right|_{E=E_{F}} \\
& =G_{0} \sum_{n} \frac{\Gamma_{L}^{(n)} \Gamma_{R}^{(n)}}{\left(\epsilon_{0}^{(n)}\right)^{2}+\left(\Gamma_{L}^{(n)}+\Gamma_{R}^{(n)}\right)^{2} / 4}, \\
S & =-\left.S_{0} \frac{\partial_{E} \tau(E)}{\tau(E)}\right|_{E=E_{F}} \\
& =-S_{0} \sum_{n} \frac{2 \epsilon_{0}^{(n)} \Gamma_{L}^{(n)} \Gamma_{R}^{(n)}}{\left[\left(\epsilon_{0}^{(n)}\right)^{2}+\left(\Gamma_{L}^{(n)}+\Gamma_{R}^{(n)}\right)^{2} / 4\right]^{2}} / G,
\end{aligned}
$$

with $G_{0}=2 e^{2} / h$ and $S_{0}=\pi^{2} k_{B}^{2} T / 3 e$.

During the stretching of a contact the atomic positions change, causing related variations in the electronic structure and in the quantum interference pattern. Therefore, we consider the parameters $\epsilon_{0}^{(n)}, \Gamma_{L}^{(n)}, \Gamma_{R}^{(n)}$ to be independent random variables distributed with probability densities $P_{\epsilon}\left(\epsilon_{0}^{(n)}\right)$, $P_{\Gamma}\left(\Gamma_{L}^{(n)}\right), P_{\Gamma}\left(\Gamma_{R}^{(n)}\right)$, respectively. For the discussion of the fluctuations of the thermopower, we determine the standard deviation,

$$
\sigma_{S}(G)=\sqrt{\left\langle S^{2}\right\rangle_{G}-\langle S\rangle_{G}^{2}}
$$

where \langle\rangle$_{G}$ means a conditional average over all configurations yielding the conductance $G$. For a system of $N$ levels the configurations are labeled by $x=$ $\left(\epsilon_{0}^{(1)}, \Gamma_{L}^{(1)}, \Gamma_{R}^{(1)}, \ldots, \epsilon_{0}^{(n)}, \Gamma_{L}^{(n)}, \Gamma_{R}^{(n)}\right)$. By introducing the probability density,

$$
p(x)=\prod_{n=1}^{N} P_{\epsilon}\left(\epsilon_{0}^{(n)}\right) P_{\Gamma}\left(\Gamma_{L}^{(n)}\right) P_{\Gamma}\left(\Gamma_{R}^{(n)}\right),
$$
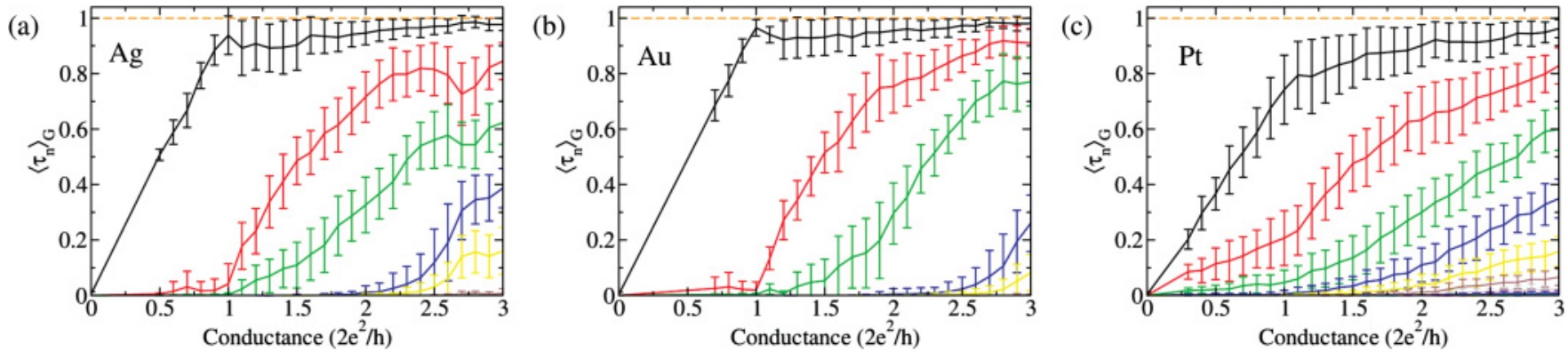

FIG. 9. (Color online) Average eigenchannel transmission probabilities $\left\langle\tau_{n}\right\rangle_{G}$ as a function of the conductance for (a) Ag, (b) Au, and (c) Pt, as determined from the atomistic simulations presented in the manuscript. Error bars show the standard deviation and the dashed horizontal lines indicate unit average transmission. See also Refs. 31 and 32 for further explanations. 

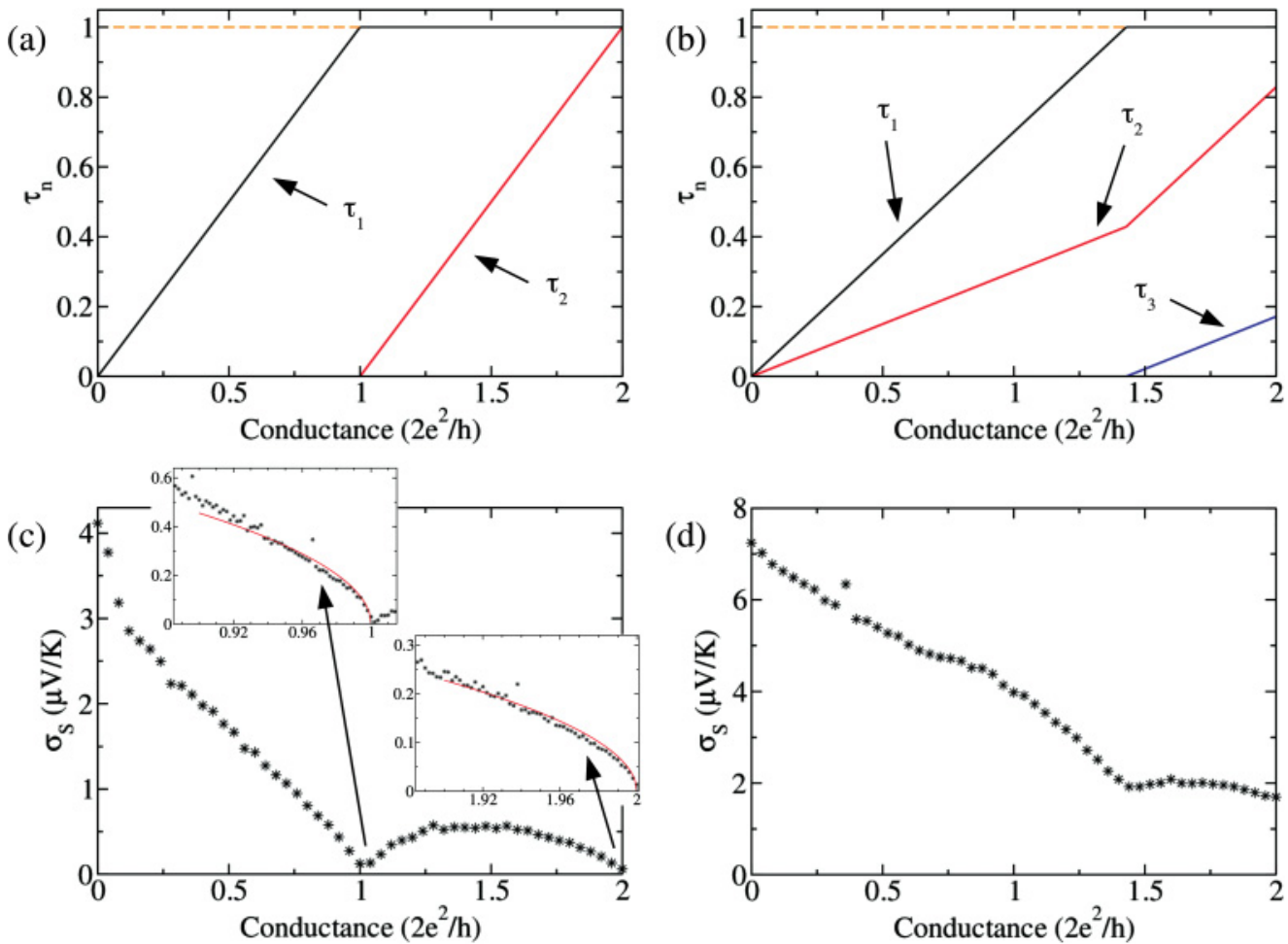

FIG. 10. (Color online) Behavior of the eigenchannel transmission probabilities $\tau_{n}$ and the standard deviation of the thermopower $\sigma_{S}$ as a function of the conductance for (a) and (c) a monovalent metal, and (b) and (d) a multivalent metal. For (c) and (d) we used $\gamma=0.6 \mathrm{eV}$ and $0.1 \mathrm{eV}$, respectively, and the bin size was set to $\Delta G=0.04 G_{0}$ in the main panels. In the insets of panel (c) we have chosen a smaller bin size of $\Delta G=0.002 G_{0}$ to resolve the selected region more clearly and show the function $\sigma_{S}=\alpha \sqrt{n-G / G_{0}} / n$ for $G \lesssim n G_{0}$ and $n=1,2$ as a red line, assuming $\alpha=1.44 \mu \mathrm{V} / \mathrm{K}$.

we can express such an average as

$$
\langle S\rangle_{G}=\int d x S(x) p(x \mid G),
$$

with the conditional probability density $p(x \mid G)=\delta(G-$ $G(x)) p(x) / \int d x \delta(G-G(x)) p(x)$. Here, $G(x)$ and $S(x)$ are determined from Eqs. (C1) and (C2), respectively.

In order to describe material-specific properties and to understand the behavior of $\sigma_{S}$ in Fig. 5, we need to know how the individual resonances $\tau_{n}\left(E, \epsilon_{0}^{(n)}, \Gamma_{L}^{(n)}, \Gamma_{R}^{(n)}\right)$ of the eigenchannels in Eq. (5) contribute to the transmission for a given conductance. For this purpose, we identify $\tau_{n}\left(E_{F}, \epsilon_{0}^{(n)}, \Gamma_{L}^{(n)}, \Gamma_{R}^{(n)}\right)$ with the measurable eigenchannel transmission probability $\tau_{n}$ to complete the ESLM. This identification procedure will be explained further in the next subsections.

\section{Monovalent metals}

As visible from Fig. 9, the saturation of channel transmission is well fulfilled in our simulations only for $G \leqslant 1 G_{0}$. However, to compare to the literature ${ }^{18,63}$ and since we are mainly interested in conductance values around $1 G_{0}$, we adopt the following ideal model to describe the monovalent metals. For a given $G$ with $n-1 \leqslant G / G_{0} \leqslant n$, we determine the transmission as

$$
\tau(E)=n-1+\tau_{n}\left(E, \epsilon_{0}^{(n)}, \Gamma_{L}^{(n)}, \Gamma_{R}^{(n)}\right) .
$$

Hence, $\tau_{1}=\cdots=\tau_{n-1}=1, \tau_{n}=G / G_{0}-(n-1)$ for $n-$ $1 \leqslant G / G_{0} \leqslant n$. This behavior of the transmission probabil- ities $\tau_{n}=\tau_{n}\left(E_{F}, \epsilon_{0}^{(n)}, \Gamma_{L}^{(n)}, \Gamma_{R}^{(n)}\right)$ as a function of the conductance is visualized in Fig. 10(a).

In Fig. 5 it is seen that $\sigma_{S}$ is suppressed at $G \approx 1 G_{0}$ for $\mathrm{Ag}$ and $\mathrm{Au}$. In order to analyze this, we consider a conductance $G$ close to but smaller than $n G_{0}$ in the ESLM. For $(n-1) \leqslant$ $G / G_{0} \leqslant n$ we obtain

$$
\begin{gathered}
G / G_{0}=n-1+\frac{\Gamma_{L}^{(n)} \Gamma_{R}^{(n)}}{\left(\epsilon_{0}^{(n)}\right)^{2}+\left(\Gamma_{L}^{(n)}+\Gamma_{R}^{(n)}\right)^{2} / 4}, \\
S / S_{0}=-\frac{2 \epsilon_{0}^{(n)}}{\Gamma_{L}^{(n)} \Gamma_{R}^{(n)}} \frac{\left(G / G_{0}+1-n\right)^{2}}{G / G_{0}} .
\end{gathered}
$$

Since $\tau_{n} \approx 1$ for $G \approx n G_{0}$, Eq. $(\mathrm{C} 7)$ requires $\Gamma_{L}^{(n)} \approx \Gamma_{R}^{(n)}$ and $\epsilon_{0}^{(n)} \approx 0$. Therefore, we consider the symmetric junction model with $\Gamma=\Gamma_{L}^{(n)}=\Gamma_{R}^{(n)} \geqslant 0$ and $\epsilon_{0}=\epsilon_{0}^{(n)}$. Since we are dealing with atomic contacts, we can expect $P_{\Gamma}(\Gamma)$ to be centered at a nonvanishing, positive value, while we assume $P_{\epsilon}\left(\epsilon_{0}\right)$ to be symmetric and maximal at $\epsilon_{0}=0$. With these assumptions, it follows that

$$
\sigma_{S}^{2}=\left\langle S^{2}\right\rangle_{G}=4 S_{0}^{2}\left\langle\frac{1}{\Gamma^{2}}\right\rangle_{G} \frac{\left(G / G_{0}+1-n\right)^{3}}{\left(G / G_{0}\right)^{2}}\left(n-G / G_{0}\right),
$$

where $\left\langle 1 / \Gamma^{2}\right\rangle_{G}$ approaches a fixed value in the limit $G \rightarrow$ $n G_{0}$. From the expression, we hence obtain Eq. (6). 


\section{Multivalent metals}

Based on the results in Fig. 9(c), we want to construct an ESLM for the multivalent Pt. To simplify the situation, we consider only two partially open channels and write

$$
\tau(E)= \begin{cases}\tau_{1}\left(E, \epsilon_{0}^{(1)}, \Gamma_{L}^{(1)}, \Gamma_{R}^{(1)}\right)+\tau_{2}\left(E, \epsilon_{0}^{(2)}, \Gamma_{L}^{(2)}, \Gamma_{R}^{(2)}\right) & \text { if } \quad 0 \leqslant G / G_{0} \leqslant 1 / a_{1}, \\ 1+\tau_{2}\left(E, \epsilon_{0}^{(2)}, \Gamma_{L}^{(2)}, \Gamma_{R}^{(2)}\right)+\tau_{3}\left(E, \epsilon_{0}^{(3)}, \Gamma_{L}^{(3)}, \Gamma_{R}^{(3)}\right) & \text { if } \quad 1 / a_{1}<G / G_{0} \leqslant\left(2 a_{1}-a_{2}\right) / a_{1}^{2},\end{cases}
$$

with $\tau_{1}=a_{1} G / G_{0}, \tau_{2}=a_{2} G / G_{0}$ for $0 \leqslant G / G_{0} \leqslant 1 / a_{1}$, and $\tau_{1}=1, \quad \tau_{2}=a_{2} / a_{1}+a_{1}\left(G / G_{0}-1 / a_{1}\right), \quad \tau_{3}=a_{2}\left(G / G_{0}-\right.$ $\left.1 / a_{1}\right)$ for $1 / a_{1}<G / G_{0} \leqslant\left(2 a_{1}-a_{2}\right) / a_{1}^{2}$. The model requires $a_{1}+a_{2}=1$, and $a_{1}=0.7$ seems to be a reasonable choice for $\mathrm{Pt}$. The upper bound for the conductance in Eq. (C10) considers in each case the full opening of a channel, and the model can easily be extended to include further partially open channels or describe larger values of $G$. The behavior of the eigenchannel transmission probabilities as a function of the conductance is visualized in Fig. 10(b).

We note that in the conductance range $0 \leqslant G / G_{0} \leqslant$ $1 / a_{1}$, for a given realization $\epsilon_{0}^{(1)}, \Gamma_{L}^{(1)}, \Gamma_{R}^{(1)}$ yielding $\tau_{1}=$ $\tau_{1}\left(E_{F}, \epsilon_{0}^{(1)}, \Gamma_{L}^{(1)}, \Gamma_{R}^{(1)}\right)$, we guess values for $\epsilon_{0}^{(2)}, \Gamma_{L}^{(2)}, \Gamma_{R}^{(2)}$ until $(1-p) a_{2} \tau_{1} / a_{1} \leqslant \tau_{2} \leqslant(1+p) a_{2} \tau_{1} / a_{1}$ with $\tau_{2}=$ $\tau_{2}\left(E_{F}, \epsilon_{0}^{(2)}, \Gamma_{L}^{(2)}, \Gamma_{R}^{(2)}\right)$ and with a small tolerance parameter $p=0.05$. For conductance values $G / G_{0}>1 / a_{1}$ we proceed similarly.

\section{Statistical analysis of the fluctuations of the thermopower}

By treating the parameters $\epsilon_{0}^{(n)}, \Gamma_{L}^{(n)}, \Gamma_{R}^{(n)}$ as independent random numbers with $\left|\epsilon_{0}^{(n)}\right| \leqslant 0.1 \mathrm{eV}$ and $0 \leqslant \Gamma_{L}^{(n)}, \Gamma_{R}^{(n)} \leqslant \gamma$, we determine $(G, S)$ pairs from the prescriptions in Eqs. (C6) and (C10). We choose a bin size $\Delta G$ and perform the typical statistical analysis. Specifically, we use $\langle S\rangle_{G}=\sum_{j} S_{j} / M$ where $S_{j}$ are all those thermopower values of the pairs with $G-\Delta G / 2 \leqslant G_{j} \leqslant G+\Delta G / 2$, and $M$ is the total number of such pairs. Analogously, we obtain the standard deviation of the thermopower as $\sigma_{S}=\sqrt{\sum_{j}\left(S_{j}-\langle S\rangle_{G}\right)^{2} / M}$.

By assuming $\epsilon_{0}^{(n)}$ to vary symmetrically around zero, we obtain $\langle S\rangle_{G} \approx 0$ in the ESLM. In this way we cannot describe the systematic deviations from zero with a unique sign, predicted and measured for quantum point contacts realized in a two-dimensional electron gas. ${ }^{20-22}$ However, our assumption is consistent with the experiments on atomic contacts, where $\langle S\rangle_{G}$ was found to scatter around zero largely within the $\sigma_{S}{ }^{18,19}$

From the numerical analysis we obtain the $\sigma_{S}(G)$ curves shown in Figs. 10(c) and 10(d). In order to obtain standard deviations comparable to the atomistic simulations we have set the maximum of the couplings to $\gamma=0.6 \mathrm{eV}$ for the monovalent metal. Because of the sharper resonances due to $d$ states for Pt (compare Figs. 3, 7, and 9 in Ref. 32) a smaller value $\gamma=0.1 \mathrm{eV}$ is needed for the model of the multivalent metal. Based on Eq. (C9) we observe that a smaller $\gamma$ should generally increase $\sigma_{S}$. To obtain ESLM results for $\sigma_{S}$ of the same size as in the experiments on $\mathrm{Au},{ }^{18} \gamma$ should correspond to around $0.2 \mathrm{eV}$. Since we would expect line widths $\Gamma_{L}^{(n)}, \Gamma_{R}^{(n)}$ around $1 \mathrm{eV}$ for atomic levels, the small values of even the maximum couplings are consistent with the interpretation that the resonances $\tau_{n}\left(E, \epsilon_{0}^{(n)}, \Gamma_{L}^{(n)}, \Gamma_{R}^{(n)}\right)$ are not simply due to atomic orbitals in the narrowest part of the contact, but that they arise essentially from disorder-related quantum interference effects.

A clear suppression of the fluctuations of the thermopower is visible in the regions $G \approx n G_{0}$ in Fig. 10(c) for the model of a monovalent metal. The insets of that panel illustrate that $\sigma_{S}=$ $\alpha \sqrt{n-G / G_{0}} / n$ at $G \approx n G_{0}$, as expected from Eq. (6). The suppression at $1 G_{0}$ is consistent with the results of our atomistic simulations and the experiments. ${ }^{18}$ For $\mathrm{Ag}$ and Au the dip at $1 G_{0}$ in Figs. 5(a) and 5(b) is reduced in depth as a result of the incomplete opening of the first conductance channel, the small contribution of further partially open conduction channels [see Figs. 9(a) and 9(b)], and the limited statistics with the related large $\Delta G=0.1 G_{0}$. These effects are enhanced at the higher conductance values, where no suppression of $\sigma_{S}$ at $2 G_{0}$ is visible in Figs. 5(a) and 5(b). Also in the experiment ${ }^{18}$ only shallow minima have been observed at the positions $n G_{0}$ for $n \geqslant 2$.

For the ESLM of a multivalent metal shown in Fig. 10(d), the first transmission channel is fully open only at $G=$ $G_{0} / a_{1} \approx 1.43 G_{0}$, so that a possible suppression of the fluctuations of $S$ is shifted to conductance values above $1 G_{0}$. However, the contribution of the second partially open channel in the ESLM, and possibly further channels in the experiments [see also Fig. 9(c)], strongly washes out the expected minimum at $G_{0} / a_{1}$. Together with the incomplete opening of the dominant conduction channel [see Fig. 9(c)] these effects explain the absence of any clear minima of $\sigma_{S}$ for the atomistic simulations in Fig. 5(c).

\footnotetext{
*fabian.pauly@kit.edu

${ }^{1}$ R. Venkatasubramanian, E. Siivola, T. Colpitts, and B. O'Quinn, Nature (London) 413, 597 (2001).

${ }^{2}$ A. I. Boukai, Y. Bunimovich, J. Tahir-Kheli, J.-K. Yu, W. A. Goddard III, and J. R. Heath, Nature (London) 451, 168 (2008).
}

${ }^{3}$ A. I. Hochbaum, R. Chen, R. D. Delgado, W. Liang, E. C. Garnett, M. Najarian, A. Majumdar, and P. Yang, Nature (London) 451, 163 (2008).

${ }^{4}$ A. I. Hochbaum and P. Yang, Chem. Rev. 110, 527 (2010).

${ }^{5}$ J. A. Malen, S. K. Yee, A. Majumdar, and R. A. Segalman, Chem. Phys. Lett. 491, 109 (2010). 
${ }^{6}$ D. Nozaki, H. Sevinçli, W. Li, R. Gutiérrez, and G. Cuniberti, Phys. Rev. B 81, 235406 (2010).

${ }^{7}$ Y. Dubi and M. Di Ventra, Rev. Mod. Phys. 83, 131 (2011).

${ }^{8}$ G. D. Mahan and J. O. Sofo, Proc. Natl. Acad. Sci. USA 93, 7436 (1996).

${ }^{9}$ P. Reddy, S.-Y. Jang, R. A. Segalman, and A. Majumdar, Science 315, 1568 (2007).

${ }^{10}$ N. W. Ashcroft and N. D. Mermin, Solid State Physics (Cengage Learning Services, Florence, KY, 1976).

${ }^{11}$ K. Baheti, J. A. Malen, P. Doak, P. Reddy, S.-Y. Jang, T. D. Tilley, A. Majumdar, and R. A. Segalman, Nano Lett. 8, 715 (2008).

${ }^{12}$ J. A. Malen, P. Doak, K. Baheti, T. D. Tilley, R. A. Segalman, and A. Majumdar, Nano Lett. 9, 1164 (2009).

${ }^{13}$ F. Pauly, J. K. Viljas, and J. C. Cuevas, Phys. Rev. B 78, 035315 (2008).

${ }^{14}$ S.-H. Ke, W. Yang, S. Curtarolo, and H. U. Baranger, Nano Lett. 9, 1011 (2009).

${ }^{15}$ A. Tan, J. Balachandran, S. Sadat, V. Gavini, B. D. Dunietz, S.-Y. Jang, and P. Reddy, J. Am. Chem. Soc. 133, 8838 (2011).

${ }^{16}$ D. Segal, Phys. Rev. B 72, 165426 (2005).

${ }^{17}$ J. K. Viljas, F. Pauly, and J. C. Cuevas, Phys. Rev. B 77, 155119 (2008).

${ }^{18}$ B. Ludoph and J. M. van Ruitenbeek, Phys. Rev. B 59, 12290 (1999).

${ }^{19}$ B. Ludoph, Ph.D. thesis, Universiteit Leiden, 1999.

${ }^{20}$ L. W. Molenkamp, H. van Houten, C. W. J. Beenakker, R. Eppenga, and C. T. Foxon, Phys. Rev. Lett. 65, 1052 (1990).

${ }^{21}$ H. van Houten, L. W. Molenkamp, C. W. J. Beenakker, and C. T. Foxon, Semicond. Sci. Technol. 7, B215 (1992).

${ }^{22}$ P. Streda, J. Phys. Condens. Matter 1, 1025 (1989).

${ }^{23}$ E. N. Bogachek, A. G. Scherbakov, and U. Landman, Phys. Rev. B 54, R11094 (1996).

${ }^{24}$ I. A. Kokurin, V. A. Margulis, and A. V. Shorokhov, J. Phys. Condens. Matter 16, 8015 (2004).

${ }^{25}$ B. J. van Wees, H. van Houten, C. W. J. Beenakker, J. G. Williamson, L. P. Kouwenhoven, D. van der Marel, and C. T. Foxon, Phys. Rev. Lett. 60, 848 (1988).

${ }^{26}$ J. C. Cuevas, A. Levy Yeyati, and A. Martín-Rodero, Phys. Rev. Lett. 80, 1066 (1998)

${ }^{27}$ E. Scheer, N. Agraït, J. C. Cuevas, A. L. Yeyati, B. Ludoph, A. Martin-Rodero, G. R. Bollinger, J. M. van Ruitenbeek, and C. Urbina, Nature (London) 394, 154 (1998).

${ }^{28}$ B. Ludoph and J. M. van Ruitenbeek, Phys. Rev. B 61, 2273 (2000).

${ }^{29}$ M. Brandbyge, J.-L. Mozos, P. Ordejón, J. Taylor, and K. Stokbro, Phys. Rev. B 65, 165401 (2002).

${ }^{30}$ N. Agraï, A. L. Yeyati, and J. M. van Ruitenbeek, Phys. Rep. 377, 81 (2003).

${ }^{31}$ M. Dreher, F. Pauly, J. Heurich, J. C. Cuevas, E. Scheer, and P. Nielaba, Phys. Rev. B 72, 075435 (2005).

${ }^{32}$ F. Pauly, M. Dreher, J. K. Viljas, M. Häfner, J. C. Cuevas, and P. Nielaba, Phys. Rev. B 74, 235106 (2006).

${ }^{33}$ F. Pauly, J. K. Viljas, U. Huniar, M. Häfner, S. Wohlthat, M. Bürkle, J. C. Cuevas, and G. Schön, New J. Phys. 10, 125019 (2008).

${ }^{34}$ Y. Dubi and M. Di Ventra, Nano Lett. 9, 97 (2009).

${ }^{35}$ Y. Dubi and M. Di Ventra, Phys. Rev. B 79, 115415 (2009).

${ }^{36}$ B. Wang, Y. Xing, L. Wan, Y. Wei, and J. Wang, Phys. Rev. B 71, 233406 (2005) [note the wrong prefactors in their Eqs. (4) and (5)].
${ }^{37}$ Y.-S. Liu, Y.-R. Chen, and Y.-C. Chen, ACS Nano 3, 3497 (2009).

${ }^{38}$ J. C. Slater and G. F. Koster, Phys. Rev. 94, 1498 (1954).

${ }^{39}$ M. J. Mehl and D. A. Papaconstantopoulos, Phys. Rev. B 54, 4519 (1996).

${ }^{40}$ M. J. Mehl and D. A. Papaconstantopoulos, Computational Materials Science (World Scientific, Singapore, 1998).

${ }^{41}$ See Supplemental Material at http://link.aps.org/supplemental/ 10.1103/PhysRevB.84.195420 for animations of the stretching processes shown in Figs. 1-3 and 6-8.

${ }^{42}$ K. W. Jacobsen, P. Stoltze, and J. K. Nørskov, Surf. Sci. 366, 394 (1996).

${ }^{43}$ M. P. Allen and D. J. Tildesley, Computer Simulation of Liquids (Oxford University Press, Oxford, 1989).

${ }^{44}$ D. Frenkel and B. Smit, Understanding Molecular Simulation: From Algorithms to Applications (Academic Press, San Diego, 2001).

${ }^{45}$ G. M. Finbow, R. M. Lynden-Bell, and I. R. McDonald, Mol. Phys. 92, 705 (1997).

${ }^{46}$ J. C. Cuevas and E. Scheer, Molecular Electronics: An Introduction to Theory and Experiment (World Scientific, Singapore, 2010).

${ }^{47}$ F. Guinea, C. Tejedor, F. Flores, and E. Louis, Phys. Rev. B 28 , 4397 (1983).

${ }^{48}$ F. Pauly, Ph.D. thesis, Universität Karlsruhe, 2007 [http://digbib.ubka.uni-karlsruhe.de/volltexte/1000007521].

${ }^{49}$ J. K. Viljas, J. C. Cuevas, F. Pauly, and M. Häfner, Phys. Rev. B 72, 245415 (2005)

${ }^{50}$ J. K. Viljas and J. C. Cuevas, Phys. Rev. B 75, 075406 (2007).

${ }^{51}$ M. Häfner, J. K. Viljas, D. Frustaglia, F. Pauly, M. Dreher, P. Nielaba, and J. C. Cuevas, Phys. Rev. B 77, 104409 (2008).

${ }^{52}$ G. Rubio-Bollinger, S. R. Bahn, N. Agraï, K. W. Jacobsen, and S. Vieira, Phys. Rev. Lett. 87, 026101 (2001).

${ }^{53}$ B. Ludoph, M. H. Devoret, D. Esteve, C. Urbina, and J. M. van Ruitenbeek, Phys. Rev. Lett. 82, 1530 (1999).

${ }^{54}$ A. I. Yanson, Ph.D. thesis, Universiteit Leiden, 2001.

${ }^{55}$ R. H. M. Smit, Y. Noat, C. Untiedt, N. D. Lang, M. C. van Hemert, and J. M. van Ruitenbeek, Nature (London) 419, 906 (2002).

${ }^{56}$ M. Kiguchi, O. Tal, S. Wohlthat, F. Pauly, M. Krieger, D. Djukic, J. C. Cuevas, and J. M. van Ruitenbeek, Phys. Rev. Lett. 101, 046801 (2008).

${ }^{57}$ R. H. M. Smit, C. Untiedt, A. I. Yanson, and J. M. van Ruitenbeek, Phys. Rev. Lett. 87, 266102 (2001).

${ }^{58}$ S. R. Bahn and K. W. Jacobsen, Phys. Rev. Lett. 87, 266101 (2001).

${ }^{59}$ V. Rodrigues, J. Bettini, A. R. Rocha, L. G. C. Rego, and D. Ugarte, Phys. Rev. B 65, 153402 (2002).

${ }^{60}$ A. I. Yanson, G. R. Bollinger, H. E. van den Brom, N. Agraï, and J. M. van Ruitenbeek, Nature (London) 395, 783 (1998).

${ }^{61}$ N. Agraï, C. Untiedt, G. Rubio-Bollinger, and S. Vieira, Phys. Rev. Lett. 88, 216803 (2002).

${ }^{62}$ J. A. Torres, J. I. Pascual, and J. J. Sáenz, Phys. Rev. B 49, 16581 (1994).

${ }^{63}$ H. E. van den Brom and J. M. van Ruitenbeek, Phys. Rev. Lett. 82, 1526 (1999). 
${ }^{64}$ H. Ohnishi, Y. Kondo, and K. Takayanagi, Nature (London) 395, 780 (1998).

${ }^{65}$ C. Untiedt, G. Rubio Bollinger, S. Vieira, and N. Agraï, Phys. Rev. B 62, 9962 (2000).

${ }^{66}$ T. Frederiksen, M. Brandbyge, N. Lorente, and A.-P. Jauho, Phys. Rev. Lett. 93, 256601 (2004).
${ }^{67}$ B. C. Hsu, Y.-S. Liu, S. H. Lin, and Y.-C. Chen, Phys. Rev. B 83, 041404 (2011)

${ }^{68}$ É. N. Bogachek, I. O. Kulik, A. N. Omel'yanchuk, and A. G. Shkorbatov, JETP Lett. 41, 633 (1985).

${ }^{69}$ O. I. Shklyarevskii, A. G. M. Jansen, J. G. H. Hermsen, and P. Wyder, Phys. Rev. Lett. 57, 1374 (1986). 